\title{
Supermassive black holes in the early universe
}

\author{
M. Dietrich ${ }^{1}$ and F. Hamann ${ }^{2}$ \\ ${ }^{1}$ Georgia State University, Department of Physics \& Astronomy, Atlanta, GA 30303, USA \\ email: dietrich@chara.gsu.edu \\ ${ }^{2}$ University of Florida, Department of Astronomy, Gainesville, FL 32611, USA \\ email: hamann@astro.ufl.edu
}

\begin{abstract}
We analyzed spectra of 15 luminous high-redshift quasars $(3.3<z<5.1)$ and we derived black hole masses that are based on $\mathrm{H} \beta$, MgII, and CIV emission lines, in the range of $2 \times 10^{8}<M_{b h}<4 \times 10^{10} M_{\odot}$. Assuming exponential growth of the black holes we estimated that it takes about several 100 Myr up to 1 Gyr to accumulate the observed black hole masses, i.e., most of the SMBHs that power these quasars at high-redshifts, started to grow at $z \simeq 5$ to 10 .
\end{abstract}

\section{Introduction}

It has been established that almost all massive galaxies are hosting a super-massive black hole $(\mathrm{SMBH})$ in their centers and that the SMHB mass is well correlated with the host galaxy's spheroidal component mass and with the bulge luminosity (Kormendy \& Richstone 1995; Magorrian et al. 1998; McLure \& Dunlop 2001). A joint galaxy and $\mathrm{SMBH}$ formation is further supported by a close correlation of the SMBH mass and with stellar velocity dispersion for both, active and non-active galaxies (Gebhardt et al. 2000; Merritt \& Ferrarese 2001; Tremaine et al. 2002). However, it is still under debate whether the black hole and the host galaxy formed simultaneously or whether one component formed before the other (Silk \& Rees 1998; Salucci et al. 1999; Kauffmann \& Haehnelt 2000; Di Matteo et al. 2003; Granato et al. 2004).

\section{Results and Discussion}

We have observed a sample of 15 luminous high-redshift quasars $(3.3 \leqslant z \leqslant 5.1$; Dietrich et al. 2002,2003a) to study the UV and optical FeII emission strength and to measure the emission line profile width of $\mathrm{H} \beta, \operatorname{MgII} \lambda 2798$, and $\operatorname{CIV} \lambda 1549$. We analyzed these quasar spectra using a multi-component fit approach (power-law continuum, FeII emission, Balmer continuum emission). We applied the equations given by Kaspi et al. (2000), McLure \& Jarvis (2002), Vestergaard (2002), and Warner et al. (2003) to estimate the black hole mass and to compare, for the first time, results based on CIV, MgII, and $\mathrm{H} \beta$. While CIV is a high-ionization line, $\mathrm{H} \beta$ and $\mathrm{MgII}$ are low-ionization lines but it can be assumed that the low and high ionization broad line gas are in gravitationally dominated motion (Onken \& Peterson 2002). The black hole masses that we determined are in the range of $2 \times 10^{8} \leqslant \mathrm{M}_{b h} \leqslant 4 \times 10^{10} M_{\odot}$ for the high luminous quasars in our sample. Within the uncertainties the $\mathrm{H} \beta, \mathrm{MgII}$, and CIV emission lines provide consistent SMBH mass estimates for each studied high redshift quasar (Fig. 1).

The presence of SMBHs at high-redshifts and the close relation of quasar activity and galaxy formation provide valuable constraints on the epoch when these SMBHs had to start to grow. For each of the high-redshift quasars we calculated a mean SMBH mass 


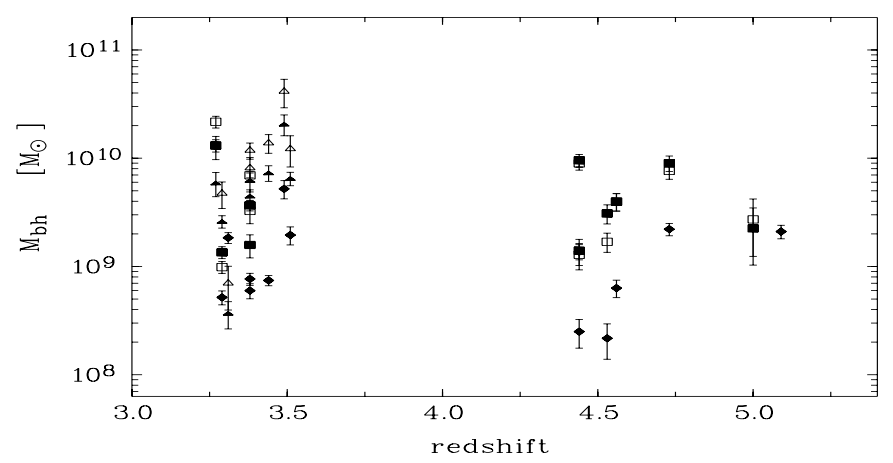

Figure 1. Estimated SMBH masses for the high-z quasars as a function of redshift $-\mathrm{H} \beta$ : open triangles (Kaspi et al. 2000), filled triangles (McLure \& Jarvis 2002); CIV: open squares (Warner et al. 2003), filled squares (Vestergaard 2002); MgII: filled diamonds (McLure \& Jarvis 2002).

based on $\mathrm{H} \beta$, MgII, and CIV. Assuming exponential growth of the black holes (e.g., Haiman \& Loeb 2001) we estimated that it takes about several $100 \mathrm{Myr}$ up to $\sim 1 \mathrm{Gyr}$ to accumulate the observed mean black hole mass. Most of the SMBH powering quasar activity at high-redshifts, started to grow at $z \simeq 5$ to 10. A delayed quasar activity with respect to a preceding vigorous star formation epoch (e.g., Archibald et al. 2002) which is indicated by high quasar gas metallicity (Dietrich et al. 2003b) provides evidence that those quasars reside in already mature host galaxies, at least with an evolved massive central component (Hamann et al. 2004).

\section{Acknowledgements}

This work was supported by grants NSF AST99-84040, and STScI AR-07988.01-96A.

\section{References}

Archibald, E. N., et al. 2002, MNRAS, 336, 353

Dietrich, M., Appenzeller, I., Vestergaard, M., \& Wagner, S. J. 2002, ApJ, 564, 581

Dietrich, M., Hamann, F., Appenzeller, I., \& Vestergaard, M. 2003a, ApJ, 596, 817

Dietrich, M., et al. 2003b, ApJ, 589, 722

Di Matteo, T., Croft, R. A. C., Springel, V., \& Hernquist, L. 2003, ApJ, 593, 56

Gebhardt, K., et al. 2000, ApJ, 543, L5

Granato, G. L., De Zotti, G., Silva, L., Bressan, A., \& Danese, L. 2004, ApJ, 600, 580

Haiman, Z., \& Loeb, A. 2001, ApJ, 552, 459

Hamann, F., Dietrich, M., Sabra, B., \& Warner, C. 2004, in Origin and Evolution of the Elements, (eds. A. McWilliams \& M. Rauch) Carnegie Obs. Astro. Ser. IV, Cambridge: CUP

Kauffmann, G. \& Haehnelt, M. G. 2000, MNRAS, 311, 576

Kaspi, S., et al. 2000, ApJ, 533, 631

Kormendy, J., \& Richstone, D. 1995, ARA\&A 33, 581

Magorrian, J., Tremaine, S., \& Richstone, D. 1998, AJ, 115, 2285

McLure, R. J., \& Dunlop, J. S. 2001, MNRAS, 327, 199

McLure, R. J., \& Jarvis, M. J. 2002, MNRAS, 337, 109

Merritt, D., \& Ferrarese, L. 2001, ApJ, 547, 140

Onken, C. A., \& Peterson, B. M. 2002, ApJ, 572, 746

Salucci, P., Szuszkiewicz, E., Monaco, P., \& Danese, L. 1999, MNRAS, 307, 637

Silk, J., \& Rees, M.J. 1998, A\&A, 331, L1

Tremaine, S., et al. 2002, ApJ, 574, 740

Vestergaard, M. 2002, ApJ, 571, 733

Warner, C., Hamann, F., \& Dietrich, M. 2003, ApJ, 596, 72 